\title{
Schlanke Diabetiker sterben früher
}

\section{Hier steht eine Anzeige.}

\begin{abstract}
Diabetiker, die zum Zeitpunkt der Diagnose normalgewichtig sind, haben innerhalb von 15 Jahren eine höhere Mortalität als ihre übergewichtigen Leidensgenossen.
\end{abstract}

Im Rahmen von fünf Kohortenstudien sind insgesamt 2565 neu diagnostizierte Patienten, die Hälfte Frauen, im mittleren Alter von 41 bis 76 Jahren und ohne kardiovaskuläre Erkrankung durchschnittlich zehn Jahre und maximal 15 Jahre lang beobachtet worden. Im Beobachtungszeitraum starben 449 Studienteilnehmer, davon 178 an kardiovaskulären Ursachen. Patienten mit normalem BMI hatten eine deutlich höhere Sterberate als Patienten mit einem BMI über $25 \mathrm{~kg} / \mathrm{m}^{2}$ (285 versus 152 Todesfälle pro 10000 Personenjahre). Auch nach Berücksichtigung von Geschlecht, Alter und kardiovaskulären Risikofaktoren inklusive Raucherstatus und Taillenumfang blieb diese Korrelation erhalten. Studien legen nahe, dass „schlanke“ Patienten ein anderes genetisches Profil besitzen als übergewichtige Diabetiker, das möglicherweise auch mit anderen Krankheiten und einer höheren Mortalität assoziiert ist, so die Spekulation der Studienautoren.

Carnethon MR et al, JAMA 2012, 308(6):581-590

\section{Hoch die Currywurst!}

\section{Kurkumin}

\section{verhindert Diabetes}

Durch regelmäßige Einnahme von Kurkumin lässt sich verhindern, dass aus einer prädiabetischen Stoffwechsellage ein manifester Diabetes wird.

Von insgesamt 240 prädiabetischen Probanden, die innerhalb einer placebokontrollierten, randomisierten Doppelblindstudie neun Monate lang täglich $250 \mathrm{mg}$ Kurkumin oder Placebo schluckten, entwickelte in der Verumgruppe kein einziger einen Diabetes - im Vergleich zu 16,4\% der Placebopatienten. NBZ, OGTT und HbAlc fielen unter Kurkumin nach drei, sechs und neun Monaten jeweils signifikant niedriger aus als unter Placebo. Die Insulinresistenz - beurteilt mit dem HOMA-IR war ebenfalls stärker zurückgegangen. Zusätzlich lieferten höhere HOMA-beta-Werte und niedrigere C-Peptid-Spiegel Hinweise auf eine verbesserte Betazellfunktion. 